\title{
Reaction on water stress of the genotypes Hordeum vulgare $L$. at different stages of plant development
}

\author{
Bome N.A. ${ }^{*}{ }^{*}$, Kolokolova N.N. ${ }^{1}$, Tetyannikov N.V. ${ }^{2}$, Weisfeld L.I. ${ }^{3}$ \\ ${ }^{1}$ Tyumen State University, Tyumen, Russia \\ ${ }^{2}$ Federal Scientific Selection and Technology Center for Horticulture and Nursery, Moscow, Russia \\ ${ }^{3}$ Emanuel Institute of Biochemical Physics, Moscow, Russia \\ *email: bomena@mail.ru
}

Phenotyping of drought tolerance in barley was carried out in vivo according to the water-holding capacity of leaves (69 samples) and under conditions simulated with using a 16-percentage of sucrose solution (12 samples). The initial material was characterized by ecological-geographical (16 countries) and genetic (2 subspecies, 21 varieties) diversity. Against the provocative background, there was a lag in the development of shoots and roots, the length of which was $20.9 \%$ (shoots) and $19.0 \%$ (roots) less than in the control. In specimens with a less pronounced stress response (L. AHOR 2542/63, Ethiopia; C.I.10995, Peru), deep roots will allow rational use of the available moisture. Well-developed shoots (Wisconsin x 691-1, USA; Nosovsky 9, Ukraine; Arna, Kazakhstan; Local, Tajikistan; Sonnet, Sverdlovsk region), covering the soil surface, will reduce the evaporation rate. In a field experiment (2018) during the period of heading-flowering ( $0.0 \mathrm{~mm}$ of precipitation; $2.1-4.00$ degrees Celsius above the norm), genotypes are differed in the water-holding capacity of the leaves. Genotypes with high and medium resistance have a significantly larger flag leaf area; chlorophyll content was $36.8-43.8$ units spad. The average yield in the tolerant group was $227.2 \mathrm{~g} / \mathrm{m}^{2}$, in the sensitive it was significantly lower $\left(72.9-186.1 \mathrm{~g} / \mathrm{m}^{2}\right)$. Samples were identified that combine resistance to moisture deficit with high productivity (Kharkovskiy 70, Ukraine; Abyssinian 14, Netherlands; Mutant 4541, Germany). Yield depends to a greater extent on field germination of seeds $(r=0.47)$, productive tillering $(r=0.51)$, grain weight per plant $(r=0.99)$. To select forms that are resistant to water stress, the assessment must be carried out at different stages of plant development, since some genotypes tolerate drought during the germination of seedlings, but are sensitive in the heading phase and vice versa. We consider tolerant samples as promising sources of drought tolerance genes for improving modern barley cultivars. 